\title{
Evaluation of Terrain Collision Risks for Flight Style Autonomous Underwater Vehicles
}

\author{
Sophia M. Schillai and \\ Stephen R. Turnock \\ Fluid Structure Interactions Group \\ University of Southampton \\ Boldrewood Innovation Campus \\ Southampton, S016 7QF \\ Email: $\{$ sms4g13, S.R.Turnock\}@ soton.ac.uk
}

\author{
Eric Rogers \\ Electronics and Computer Science \\ University of Southampton \\ University Road, \\ Southampton, SO17 1BJ \\ Email: etar@ecs.soton.ac.uk
}

\author{
Alexander B. Phillips \\ National Oceanography Centre \\ European Way, \\ Southampton, SO14 3ZH \\ Email: abp@noc.ac.uk
}

\begin{abstract}
Photographic surveys of the seafloor with flight style autonomous underwater vehicles are a very effective tool for discovery and exploration. Due to the high terrain collision risk for the survey vehicle, they are employed with caution. The extent of this risk remains unquantified. For mission planning, researchers and vehicle operators have to rely on their experience. This paper introduces measures for vehicle risk and success and analyses how previously mapped terrains and artificially generated terrain maps can be used to categorize terrains. The developed measures are applied to a simulation of the Autosub6000 flight style AUV terrain following system. Based on quantitative parameters, changes to the obstacle avoidance system and survey mission plans can be better informed.
\end{abstract}

Index Terms-auv, terrain following, risk, photographic survey, Autosub6000.

\section{INTRODUCTION}

The ocean floor is often called the benthic zone in an ecologic context, and is home to a large number of organisms. For most of the oceans it has never been mapped, the few existing studies of temporal changes highlight that significant vairations occur, making regular mapping of larger areas a desired goal [1]. Benthic surveys may be undertaken for ground truthing sonar images, mapping of habitats, measuring sediment transports and deposition, or for establishing the location and activity of hydrothermal vents and volcanoes [2], [3]. Monitoring may be required for: research purposes, planning and maintenance of human-made structures or for determining the human impact on ecosystems [4]. In [3], Wynn et al highlight the special interest in extreme environments such as volcanoes or hydrothermal vents, both of which feature a terrain that is difficult to navigate, with obstacles that have a small cross section but bring a large change in ocean floor height.

The Autosub vehicles developed at the National Oceanography Centre in the UK regularly undertake missions where they closely follow an unknown terrain structure, for photographic surveys between $2 m$ and $4 m$ altitude, as undertaken by Autosub6000 during the cruises D343 [5] and D377 [6]. Operation close to the seafloor is required since suitable lighting needs to be provided by the AUV and turbidity in the water can obscure the image if the altitude of the camera is too high.
The scientific contributions based on photographic and sonar images and sonar data collected during benthic surveys are numerous [7], but these mission types also present an increased risk of vehicle loss, since an accidental collision with the terrain can lead to damage or even loss of the platform [8].

Following the local bathymetry at the ocean floor at a constant distance without endangering the vehicle, while ensuring image quality is the main challenge for photographic surveys. In the worst case a crash with the seafloor can lead to the vehicle gaining additional weight, either by damaging a pressure vessel or by filling the hull with mud from the seafloor. Most AUVs are slightly positively buoyant and carry emergency drop weights so in case of a technical failure they will return to the surface. Added weight from an accident counteracts these measures and can leave the vessel unable to surface. In many regions of the worlds oceans, recovery of an AUV from the seabed can be too costly to justify. The highest priority in terrain following is therefore crash free manoeuvring of the vehicle. To mitigate the consequences of a collision, Autosub6000 has fenders and a skid panel added at the front of the vehicle [5].

Terrain collision is seen as a "High impact fault" [8], but the risk of collision is usually regarded as small, since typical missions run at higher altitudes [9]. A further analysis of the factors determining the terrain collision risk has not been found. When planning a photographic survey at full ocean depth, there is usually very little information about the terrain known beforehand, since vast majorities of the oceans remain unmapped [10]. The mapping available is obtained during the research cruise from the ship or preceding sonar scans at higher altitudes, and its resolution is usually too coarse for the scale of the vehicle. A pre-analysis with path planning as conducted for aggressive terrain following in [11] is thus impossible. Judging vehicle safety and choosing safe altitude demands is often based on experience only.

This paper combines selected real terrains and test terrains with a mathematical model of Autosub6000 and a simulation based on its terrain following behaviour. The risk to the vehicle over known terrains is estimated to get a better understanding of the factors determining the collision risk. By introducing 
measures for quantifying expected vehicle risk and achievable mission success, different configurations can be compared, allowing a more informed choice of obstacle avoidance system and mission parameters.

\section{AIM AND OBJECTIVES}

To achieve the aim of being able to better understand the terrain and vehicle factors that determine mission success and risks of low altitude terrain following, the objectives of the research on which this paper is based are the following:

1) Simulate the performance of the Autosub6000 vehicle at low altitudes.

2) Introduce measures for the vehicle collision risk and photographic survey success for quantitive analysis.

3) Investigate relevant terrain parameters.

4) Choose suitable real terrains.

5) Generate suitable random terrains.

6) Simulate vehicle performance over a variety of parameters.

7) Analyse the influence of these parameters on the vehicle performance.

\section{Autosub6000 Terrain Following}

Autosub6000 uses a foward looking mechanical scanning sonar for its terrain following system. The sonar is mounted in a 90 degree rotated position and the angle of the sonar beam is continuously varied to detect the highest point of the terrain within the sonar range. Figure 2 shows the sonar and its scanning range. Depending on the detection result, the sonar increases or decreases its head angle, tracking the angle of transition between detection and no detection of the horizon. If terrain is not detected for a whole scan, the lower half of the sonar range is continuously scanned to ensure safety during descent. If terrain is detected, the range and pitch angle at the moment of detection are saved in an array, the array position represents the angle of the sonar head. Independent of a detection, the highest sonar angle with a valid entry is then used for horizon tracking. An auxiliary parameter, called the "pseudo altitude" is calculated using the sonar angle, the terrain range and the pitch angle at the moment of detection. To allow both close terrain following and steep ascends, the pseudo altitude is increased based on a threshold.

For the altitude control of the vehicle, this pseudo altitude is compared to the altitude measured by a separate altitude sensor and the lower of the two values is used to calculate the altitude error from the altitude demand. The altitude error is then translated into a depth demand for use in the depth PID control. A detailed description of the sonar scanning algorithm and pseudo altitude calculation is given in [13].

\section{TERRAin Following Simulation}

The terrain following simulation is implemented in Python, using the Robot Operating System (ROS) [14] framework. With the ROS nodes and publisher system, all data generated during a simulation run can be recorded, details about data can

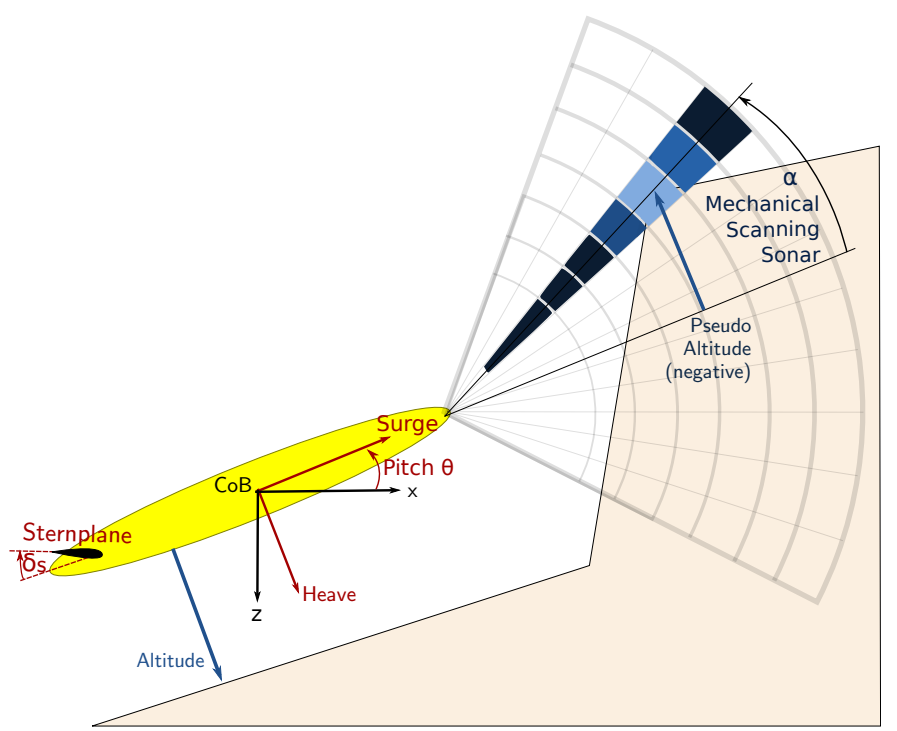

Fig. 2. Overview of the components relevant for terrain collision avoidance on the Autosub6000 AUV.

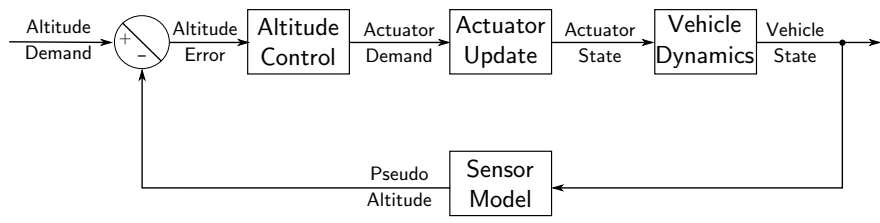

Fig. 3. Overview of the structure of the terrain following simulation.

be retrieved and components such as vehicle model or terrain following algorithms can be exchanged.

Each time step of the main simulation loop generates a sensor reading from the terrain and the current state of the vehicle, giving the control input of the altitude control. The altitude control results in an actuator update, considering physical limits of the actuators. The vehicle model takes the vehicle state and actuator setting at the start of each time step and generates the state at the next time step. The simulation is purely in the vertical plane (heave and pitch), assuming a constant forwards speed (as ensured by a separate control loop on the vehicle). The vehicle model is based on [15], considering hydrodynamic coefficients for viscous damping, added mass and radiation damping. The simulation focuses on the vertical plane, with a constant forwards velocity. Thus the coefficients $\dot{u}, v, \dot{v}, \phi, p, \dot{p}, \psi, r$, and $\dot{r}$ are assumed to be zero. 


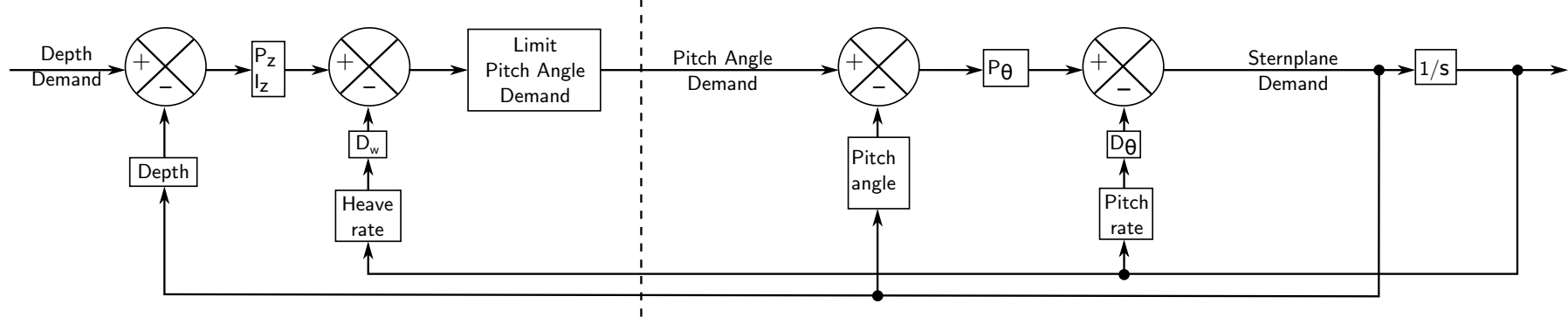

Fig. 1. Pitch PID law, from [12].

$$
\begin{aligned}
m(\dot{w}-u q) & =\sum_{i} Z_{i} \\
& =Z_{\dot{q}} \cdot \dot{q}+Z_{\dot{w}} \cdot \dot{w} \\
& +Z_{q u} \cdot q u+Z_{w u} \cdot w u \\
& +Z_{|q| w} \cdot|q| w+Z_{|w| w} \cdot|w| w \\
& +Z_{\delta_{s} u u} \cdot \delta_{s} u u \\
& +(W-B) \cdot \cos \theta \\
I_{y y} \cdot \dot{q} & =\sum_{i} M_{i} \\
& =M_{\dot{q}} \cdot \dot{q}+M_{\dot{w}} \cdot \dot{w} \\
& +M_{q u} \cdot q u+M_{w u} \cdot w u \\
& +M_{|w| q} \cdot|w| q \\
& +M_{\delta_{s} u u} \cdot \delta_{s} u u \\
& +B G \sin \theta
\end{aligned}
$$

The hydrodynamic derivatives used for Autosub6000 are taken from [16].

The depth control approach reflects the depth control of Autosub6000, utilising a cascaded PID law (figure 1), as described in [12].

The model of the horizon following sonar is implemented so the scanning rate and delays can be varied. The centre of the sonar beam is used for calculating the sonar range and the scanning algorithm and pseudo altitude calculations in the original version are as described in [13]. The calculation of the pseudo altitude and sonar range are modified to see how they could change the mission success and risk.

\section{VALIDATION}

The model stability was tested against frequency variations both for the overall system frequency and the model resolution itself. As stable parameters, a system frequency of $10 \mathrm{~Hz}$ and a model resolution of $50 \mathrm{~Hz}$ was chosen. The system speedup is limited by the maximum speed of the ROS framework, however the added features through ROS are considered a higher gain and the simulation can still be run faster than real time on a standard laptop. The model was then validated against data recorded with Autosub6000 on cruises D343 and D377. The terrain outline and vehicle path are generated based on the vehicle altitude and depth measurements during the mission. Since not all data necessary for simulation are available for the Autosub6000 configuration at the time, the main purpose of this validation is to demonstrate that the simulation can be used to make statements about terrain following for vehicles in the style of Autosub6000.

Figure 4 compares the simulated trajectory compared to a path flown during Autosub6000 mission 57. Overall the simulation is a close match to the real vehicle and thus suitable for testing changes on the terrain following and obstacle avoidance system. For steep terrain changes, the simulated vehicle has a larger error in goal altitude. This is due to the $\mathrm{x}$ axis of the terrain being approximated based on a constant forward velocity of the vehicle in the global coordinate system. During steep ascends the direction of movement of the vehicle is no longer parallel to the global $\mathrm{x}$ axis, so the real terrain slopes were less steep than they present themselves in the estimated terrain.

\section{Measures FOR Risk AND Mission Success}

The goal altitude of $3 \mathrm{~m}$ is of the same order of magnitude as the vehicle dimensions. Whilst the Centre of Gravity or the simulated sensor altitude measurements may be at a safe distance to the terrain, other parts of the vehicle may already be in contact with the terrain. In addition, a faulty sensor measurement might lead to a sudden wrong altitude demand, causing a single wrong motion of the vehicle. To understand the risk to the vehicle, its full size and freedoms of motion are considered. A bounding box is fitted around the vehicle (see figure 5). The y-direction is limited by the front and aft of the vehicle, in the $\mathrm{z}$-direction the lowest point of all vehicle components yields the limit. For each simulation step, the shortest distance between the terrain and the bounding box is determined as the collision distance altitude ${ }_{B B}$. In addition, the altitude of the centre of gravity altitude ${ }_{C o G}$ of the vehicle is monitored. Since all collision behaviours at this point would be estimates based on little experience, the collision itself is not modelled. Instead, all distances are calculated based on the normal model, to give an impression how much the vehicle path intersects with the terrain. This is used to estimate the severity of the resulting collision. The added fenders can protect Autosub6000 from minor collisions, so a collision with altitude $e_{o G}>0$ is considered less severe. If the bounding box has a positive distance to the terrain, the transition to very low risk operation is structured further, 
Terrain following results M57

Goal altitude $3.5 \mathrm{~m}$

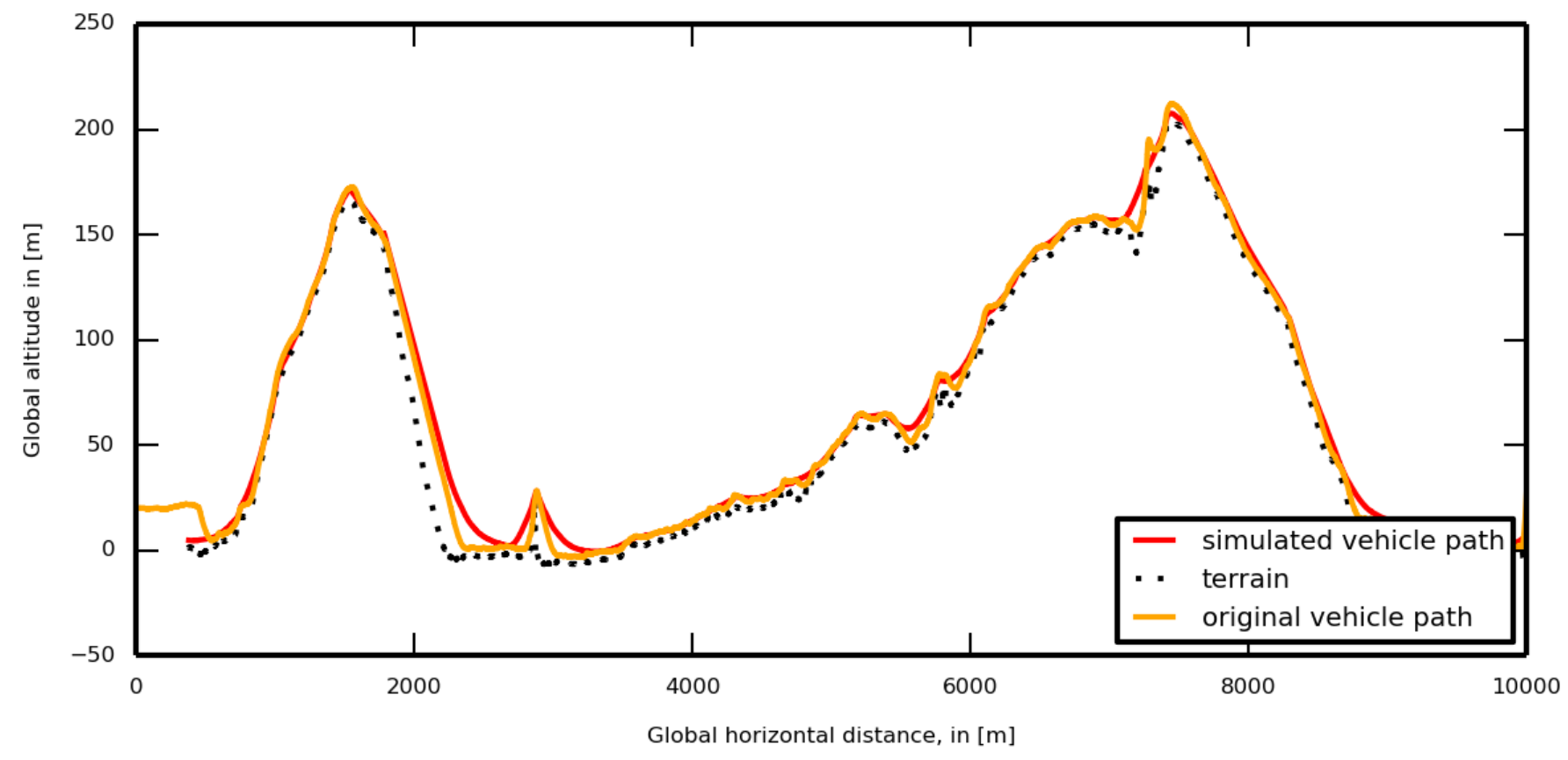

Simulated vehicle altitude error

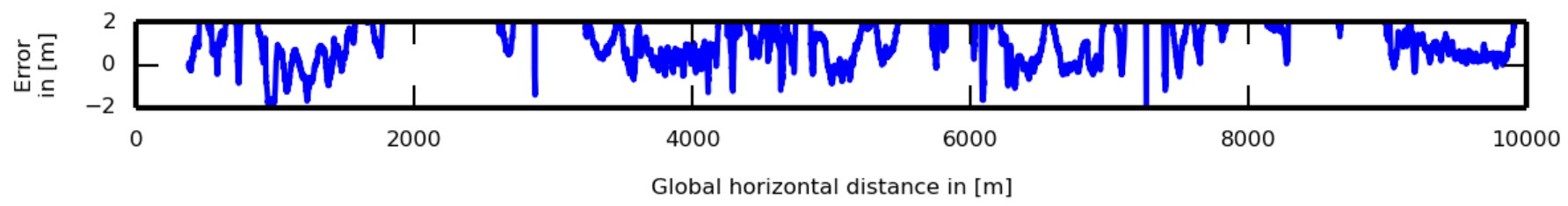

Difference between simulated and experiment altitude

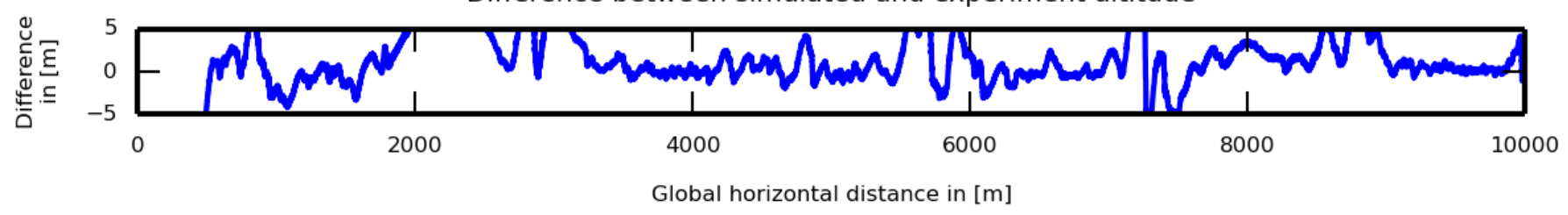

Altitude in risk zones

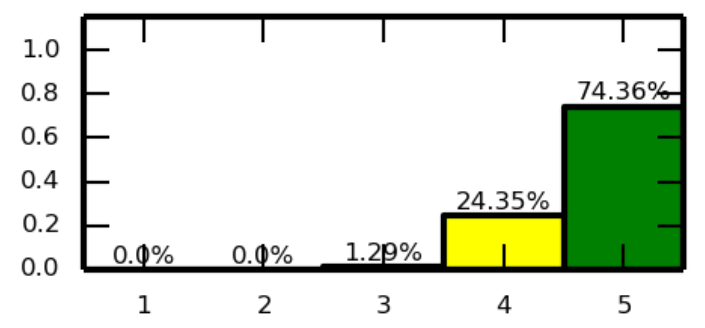

Photographic survey success

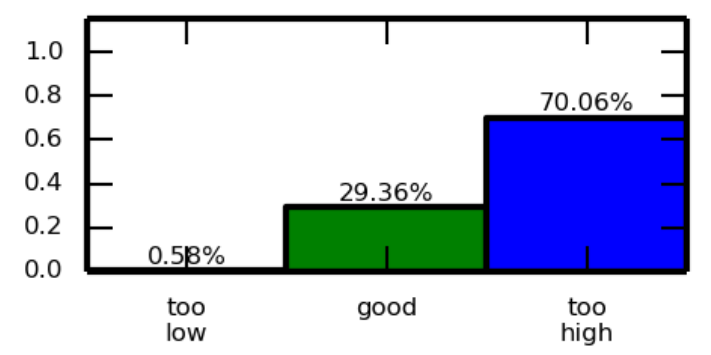

Fig. 4. Comparison to M57 mission. 


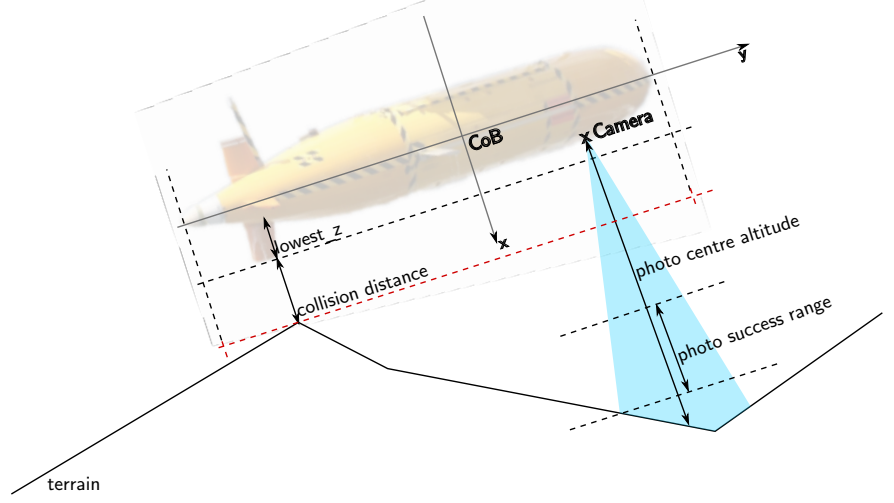

Fig. 5. Illustration of bounding box, collision distance and photo centre altitude.

based on the possible altitude change caused by a maximum or minimum pitch demand.

If the pitch changes from zero to Min. $\theta$, the distance by which the tip of the vehicle moved is estimated as lowest_tip $\approx 1.3 \mathrm{~m}$, similarly lowest_tail $\approx 2.9 \mathrm{~m}$ gives the distance by which the tail would move for a sudden pitch change from zero to Max. $\theta$. A nose collision is considered more severe, since it has a higher risk of damage that can change the buoyancy of the vehicle, whilst a tail collision may have a severe impact on the manoeuvrability but is unlikely to change buoyancy. The collision distance is therefore split into five regions:

1) altitude $_{C o G}<0$, altitude al $_{B B}<0$ : Serious collision.

2) altitude $_{C o G}>0$, altitude al $_{B}<0$ : Likely collision

3) $0<$ altitude $_{B B}<$ lowest_tip: Both tail and tip collision possible.

4) lowest_tip $<$ altitude $_{B B}<$ lowest_tail: Tail collision possible.

5) lowest_tail $<$ altitude $_{B B}$ : Collision unlikely

For mission success evaluation, the photo centre altitude is calculated as the distance between the camera and the centre of the recorded frame. The minimum collision distance is the smallest collision distance that will still be accepted as safe. The photo success range is a minimum and maximum altitude at which the photo is still considered useful. As specified in [7], the photo success range for all simulations is between $1.9 \mathrm{~m}$ and $4.2 \mathrm{~m}$, the survey success zone is therefore the area where the photo centre altitude is within the photo success range. To allow comparison of terrains, the risk quota and the success quota give the proportion of the terrain where the vehicle is in the above risk zones and the survey success zone respectively.

\section{Simulation TERRAins}

For the results presented here, three types of test terrains were used: simplified artificial, real and randomly generated terrains. The simplified artificial terrains use steps of various heights $(\propto d z)$ and slopes $\left(\propto \frac{d z}{d t}\right)$ to test the limits of the
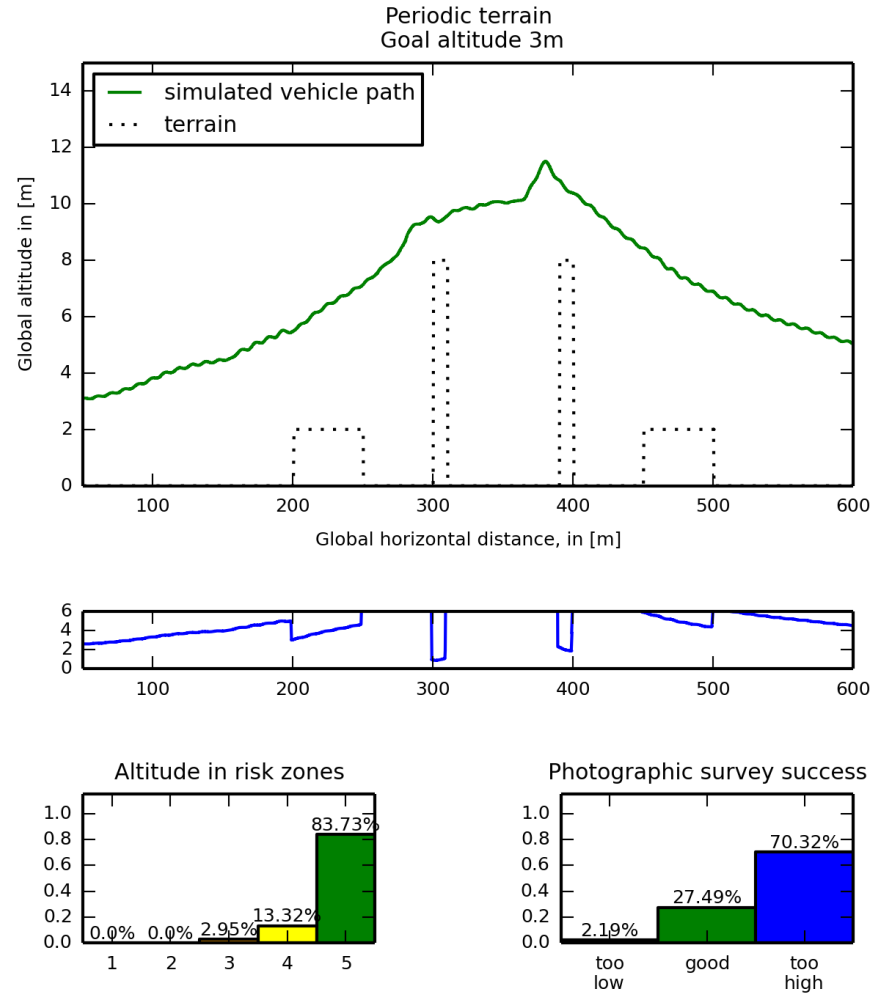

Fig. 6. Artificial terrain A.

vehicle and illustrate relevant properties of the terrain. The real terrains are terrains from the D343 and D377 cruise. To get a larger range of terrains with a controlled set of measures, random terrains were also generated.

The comparison of the two periodic terrains in figures 6 and 7 illustrates that the step height and change alone are not a sufficient description for a terrain. Two terrains with the exact same step changes can result in very different vehicle mission success. Due to the asymmetric pitch limits and the upwards force from the positive buoyancy, the vehicle risk and photographic survey success also depend on the direction. Terrain descriptions therefore need to consider directional information both in the $\mathrm{z}$ and the $\mathrm{x}$ direction.

The random terrains were generated with a $1 \mathrm{~m}$ resolution. At the start and end of each terrain, a $150 \mathrm{~m}$ section of constant altitude was added, so all terrains have similar start and end conditions. The first random generated terrains use random numbers to generate the difference between consequent terrain points. The terrain step was scaled on a slope limit $\left(\propto \frac{d z}{d x}\right)$, symmetrical in the positive and negative direction. As a second type of terrain generation, random numbers were used to generate the slope change between terrain points $\left(\propto \frac{d^{2} z}{d x^{2}}\right)$ whilst applying a maximum step limit. As an initial test set, 90 terrains of $1000 \mathrm{~m}$ length were generated with slope rates of $0.05 / m, 0.10 / m$, and $0.15 / m$. For each slope rate, slope limits of $0.5,1$ and 1.5 were applied and ten terrains were generated . The random terrains obtained this way are shown in figure 8 . The three terrains with the highest percentage in (Risk Zone 

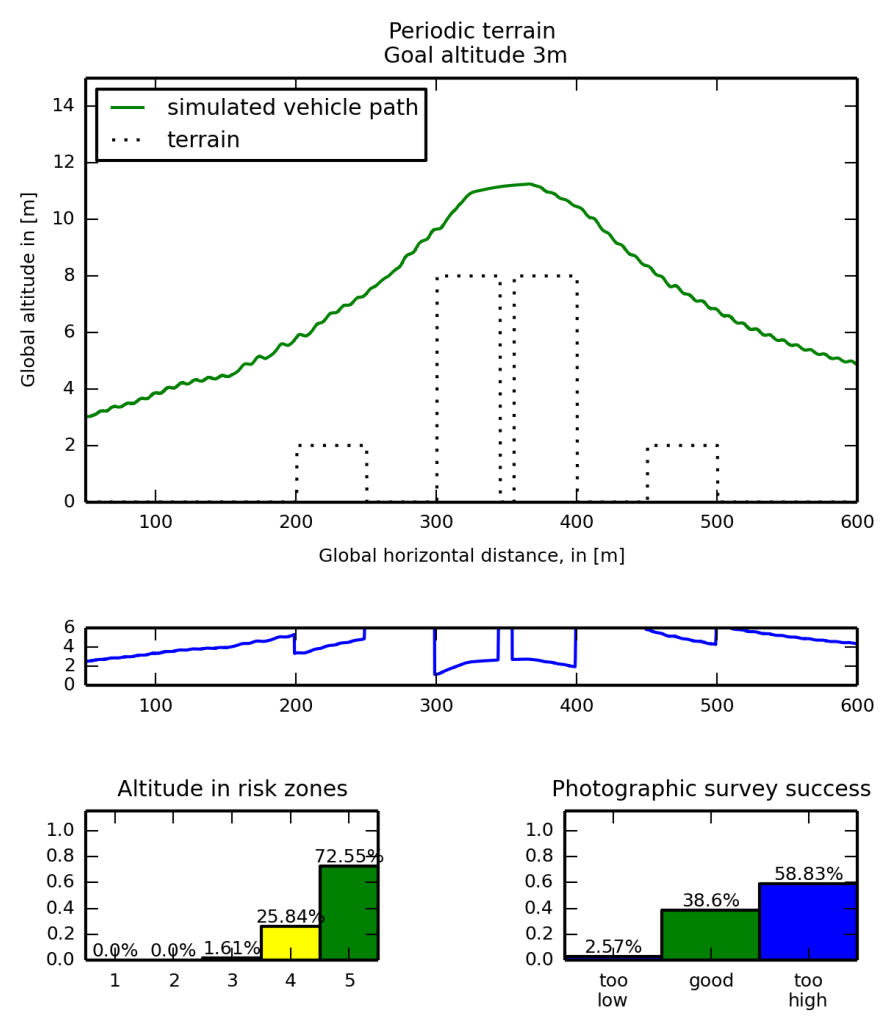

Fig. 7. Artificial terrain B.

$1+$ Risk Zone 2) and the three terrains with the worst photo success are shown in figure 9. A comparison of real terrains like in figure 4 and the random generated terrains shows, that overall terrain variations are well depicted, however sudden steep cliffs are still missing from the random terrains. The worst terrains all require the vehicle to exceed its maximum ascent rate over a distance that is larger than the sonar range, it is therefore impossible for the vehicle to avoid a crash unless it changes its path in the horizontal plane. For a benchmarking set of terrains it may be useful to exclude such features.

\section{RESULTS}

Based on the simulation results over the artificial and random terrains, the understanding of parameters for describing terrain complexity has been improved.

To see if the current terrain following algorithm would perform better with a faster mechanical scanning sonar or a multibeam sonar, the sonar was run at a frequency of $10 \mathrm{~Hz}$ rather than $1 \mathrm{~Hz}$ and without delay. For some artificial terrains small gains where observed, as the sonar would find the horizon again more quickly. Over the real and artificial terrains, a marginal reduction in the risk was found, but at the same time the vehicle altitude is forced to be more conservative, which has a slight negative impact on the photo success rate.

During the validation of the simulation it was noticed that the use of previous sonar values for generating the pseudo altitude sometimes lead to very outdated values being used. The pseudo altitude calculation was then modified. If no terrain

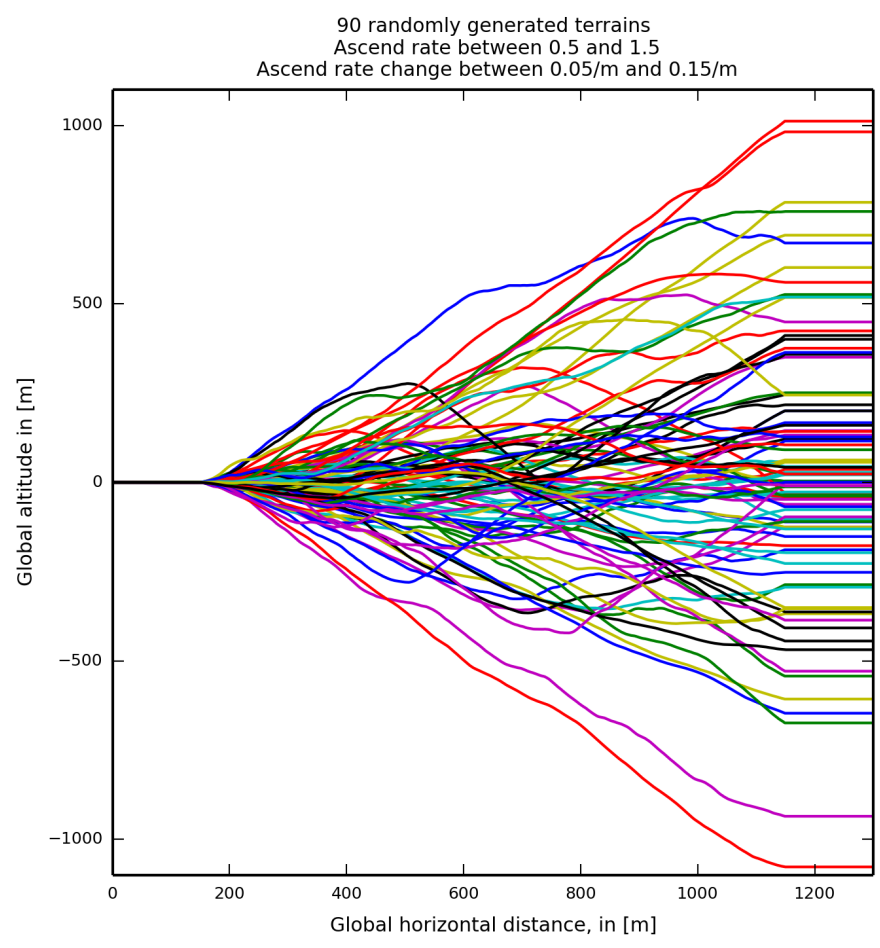

Fig. 8. Overview of all random terrains used for this paper.

has been detected, the last pseudo altitude generated from a terrain detection is used until the sonar scanned its minimum angle or terrain is detected again. This slightly improved the mission success rate, whilst only varying the vehicle risk between zones 4 and 5 .

\section{CONCLUSION}

The terrain following simulation has been shown to be suitable for testing changes to the obstacle avoidance. A clear and quantifiable description of vehicle risk and mission success has been introduced and its use has been demonstrated. Researchers planning to use photographic surveys for their research can be given an estimate how suitable a flight style vehicle will be for their terrain. Vehicle operators can find the likelihood of a terrain collision or a mission abort to avoid terrain collision.

The understanding of terrain measures will be used to compile a standard set of terrains for risk assessment and benchmarking of new obstacle avoidance algorithms. The next steps for improving the terrain following capability are now to design a mapping algorithm, which can generate a map from the limited available live data. This map can then be used for trajectory planning. With a mapping algorithm, the question of a faster sonar sensor will be asked again. For trajectory planning, questions regarding the appropriate projection of the track, the trajectory planning strategy and the integration of control into the obstacle avoidance will be analysed.

Throughout the development, the simulation and quantification described in this paper will be applied to test performance changes dependant on modifications of the sensor and obstacle avoidance configuration. 


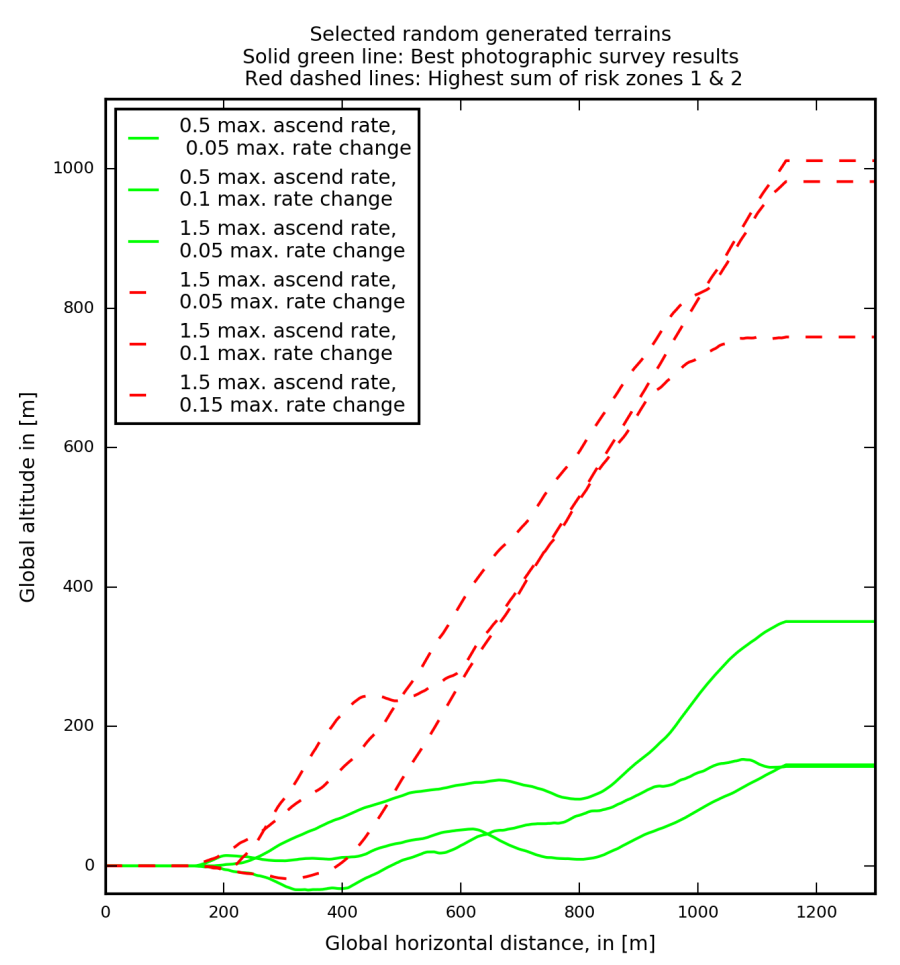

Fig. 9. Terrains with the best photographic survey results and the worst collision risk for terrain following at $3 \mathrm{~m}$ goal altitude.

\section{APPENDIX A}

\section{MODEL COEFFICIENTS}

The model coefficients for Autosub6000 are from a model test for Autosub1, using a 3/4 scale model, obtained through steady state and dynamic experiments as described in [16].

\begin{tabular}{lrl} 
Coefficient & Value $\cdot 10^{3}$ & Dimensionalisation \\
\hline$Z_{w u}^{\prime}$ & -28.45 & $1 / 2 \rho \cdot l^{2}$ \\
$Z_{\dot{w}}^{\prime}$ & -17.39 & $1 / 2 \rho \cdot l^{3}$ \\
$Z_{|w| w}^{\prime}$ & -27.05 & $1 / 2 \rho \cdot l^{2}$ \\
$Z_{q u}^{\prime}$ & -12.64 & $1 / 2 \rho \cdot l^{3}$ \\
$Z_{\dot{q}}^{\prime}$ & -0.169 & $1 / 2 \rho \cdot l^{4}$ \\
$Z_{|q| w}^{\prime}$ & -6.87 & $1 / 2 \rho \cdot l^{3}$ \\
$Z_{\delta_{s} u u}^{\prime}$ & 5.31 & $1 / 2 \rho \cdot l^{2}$ \\
$M_{w u}^{\prime}$ & 4.54 & $1 / 2 \rho \cdot l^{3}$ \\
$M_{\dot{w}}^{\prime}$ & -0.17 & $1 / 2 \rho \cdot l^{4}$ \\
$M_{q u}^{\prime}$ & -5.35 & $1 / 2 \rho \cdot l^{4}$ \\
$M_{\dot{q}}^{\prime}$ & -0.98 & $1 / 2 \rho \cdot l^{5}$ \\
$M_{|w| q}^{\prime}$ & -2.57 & $1 / 2 \rho \cdot l^{3}$ \\
$M_{\delta_{s} u u}^{\prime}$ & \multicolumn{2}{c}{} \\
\multicolumn{2}{c}{2.11} & $1 / 2 \rho \cdot l^{3}$ \\
& \multicolumn{2}{c}{ TABLE I }
\end{tabular}

OVERVIEW OF NON-DIMENSIONAL HYDRODYNAMIC COEFFICIENTS USED FOR THE VEHICLE MODEL.

\begin{tabular}{lrrc} 
Parameter & M25 & M57 & Units \\
\hline Mass & 1727.6 & 1727.6 & $\mathrm{~kg}$ \\
Length & 5.913 & 5.913 & $\mathrm{~m}$ \\
$I_{y} y$ & 4950 & 4950 & $\mathrm{~kg} \cdot \mathrm{m}^{2}$ \\
BG & 0.021 & 0.021 & $\mathrm{~m}$ \\
W - B & -151 & -151 & $\mathrm{~N}$ \\
Min. $\theta$ & -17.2 & -17.2 & degrees \\
Max. $\theta$ & 57.3 & 68.8 & degrees \\
Min. $\delta_{s}$ & 20 & 20 & degrees \\
Max. $\delta_{s}$ & 20 & 20 & degrees \\
$\delta_{s}$ change rate & 15 & 15 & degrees / s \\
Min. $\alpha$ & 30 & 30 & degrees \\
Max. $\alpha$ & 30 & 30 & degrees \\
Sonar step size & 3 & 3 & degrees \\
Sonar panic distance & 30 & 30 & $\mathrm{~m}$ \\
Sonar panic offset & 7 & $7 \mathrm{~m}$ & \\
Sonar original rate & 1 & 1 & $\mathrm{~Hz}$ \\
Sonar original delay & 2 & 2 & $\mathrm{~s}$ \\
Sonar speed up rate & 10 & 10 & $\mathrm{~Hz}$ \\
Sonar speed up delay & 0 & 0 & $\mathrm{~s}$ \\
$P_{z}$ & 0.06 & 0.15 & \\
TC $C_{z}$ & 60 & 60 & $\mathrm{~s}$ \\
$D_{z}$ & 0.1 & 0.1 & \\
Max.I & 0.5 & 0.4 & \\
Min.I & -0.05 & -0.15 & \\
$P_{\theta}$ & 2.2 & 2.2 & \\
$D_{\theta}$ & 6 & 6 & \\
& TABLE II &
\end{tabular}

LIST OF SIMULATION PARAMETERS.

\section{APPENDIX B}

\section{AutOSUb6000 SIMULATION PARAMETERS}

Core parameters and limitations used for the simulation are listed below. $\theta$ is the pitch angle, $\delta s$ the angle of the horizontal sternplanes and $\alpha$ the angle of the mechanical scanning sonar, relative to the vehicle. Angles are given in degrees for better human readability, but only radians are used in simulation.

\section{REFERENCES}

[1] A. G. Glover, A. J. Gooday, D. M. Bailey, D. S. M. Billett, P. Chevaldonné, A. Colaco, J. Copley, D. Cuvelier, D. Desbruyeres, V. Kalogeropoulou, and Others, "Temporal change in deep-sea benthic ecosystems: a review of the evidence from recent time-series studies," Advances in marine biology, vol. 58, pp. 1-95, 2010.

[2] D. W. Caress, H. Thomas, W. J. Kirkwood, R. McEwen, R. Henthorn, D. A. Clague, C. K. Paull, J. Paduan, and K. L. Maier, "High-resolution multibeam, sidescan, and subbottom surveys using the MBARI AUV D. Allan B," Marine habitat mapping technology for Alaska, pp. 47-69, 2008.

[3] R. B. Wynn, V. A. I. Huvenne, T. P. Le Bas, B. J. Murton, D. P. Connelly, B. J. Bett, H. A. Ruhl, K. J. Morris, J. Peakall, D. R. Parsons, and Others, "Autonomous Underwater Vehicles (AUVs): their past, present and future contributions to the advancement of marine geoscience," Marine Geology, vol. 352, pp. 451-468, 2014.

[4] R. Nakajima, H. Yamamoto, S. Kawagucci, Y. Takaya, T. Nozaki, C. Chen, K. Fujikura, T. Miwa, and K. Takai, "Post-Drilling Changes in Seabed Landscape and Megabenthos in a Deep-Sea Hydrothermal System, the Iheya North Field, Okinawa Trough," 2015.

[5] S. D. McPhail, "RSS Discovery Cruise 343: Deepwater trials of the Autosub6000 AUV, HyBIS, and telemetry systems," Southampton, pp. $1-25,2010$.

[6] H. A. Ruhl, "RRS Discovery Cruise 377 \& 378: Autonomous ecological surveying of the abyss: understanding mesoscale spatial heterogenety at the Porcupine Abyssal Plain,” pp. 1-191, 2013.

[7] K. J. Morris, B. J. Bett, J. M. Durden, V. A. I. Huvenne, R. Milligan, D. O. B. Jones, S. McPhail, K. Robert, D. M. Bailey, and H. A. Ruhl, "A new method for ecological surveying of the abyss using autonomous underwater vehicle photography," Limnology and Oceanography: Methods, vol. 12, pp. 795-809, 2014. 
[8] G. Griffiths and A. Trembanis, "Towards a Risk Management Process for Autonomous Underwater Vehicles," vol. 44, no. 0, 2006.

[9] M. P. Brito, "Autosub Long Range risk assessment report: expert elicitation and risk discussion record," p. 17, 2015.

[10] M. F. J. Wilson, B. O'Connell, C. Brown, J. C. Guinan, and A. J. Grehan, "Multiscale terrain analysis of multibeam bathymetry data for habitat mapping on the continental slope," Marine Geodesy, vol. 30, no. 1-2, pp. 3-35, 2007.

[11] S. E. Houts and S. M. Rock, "Trajectory planning for motion-constrained AUVs in uncertain environments," 2014 Oceans - St. John's, OCEANS 2014, 2015.

[12] M. E. Furlong, S. D. McPhail, and M. Pebody, "A New Collision Avoidance System for the Autosub6000 Autonomous Underwater Vehicle,' 2009.

[13] S. D. McPhail, M. Furlong, and M. Pebody, "Low-altitude terrain following and collision avoidance in a flight-class autonomous underwater vehicle," Proceedings of the Institution of Mechanical Engineers, Part M: Journal of Engineering for the Maritime Environment, vol. 224, no. 4, pp. 279-292, 2010. [Online]. Available: http://eprints.soton.ac.uk/169447/

[14] M. Quigley, K. Conley, B. Gerkey, J. Faust, T. Foote, J. Leibs R. Wheeler, and A. Y. Ng, "ROS: an open-source Robot Operating System," ICRA workshop on open source software, vol. 3, no. 3.2, p. 5, 2009.

[15] M. Gertler and G. R. Hagen, "Standard Equations of Motion for Submarine Simulation," Naval Ship Research and Deverlopment Center, Tech. Rep., 1967.

[16] N. I. Kimber and W. B. Marshfield, "Design and Testing of Control Surfaces for the Autosub Demonstrator Test Vehicle," 1993. 